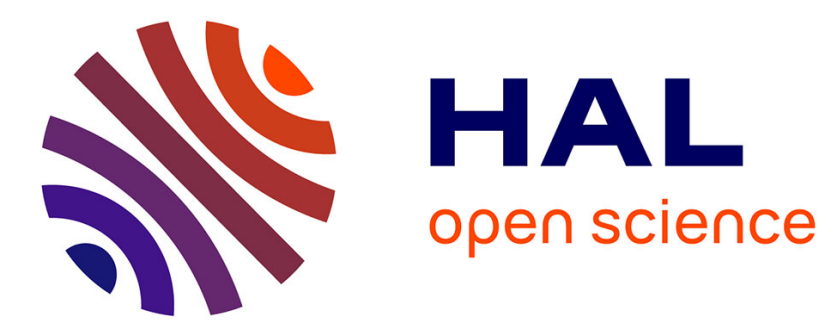

\title{
Optimal object association in the Dempster-Shafer framework
}

Thierry Denoeux, Nicole El Zoghby, Véronique Cherfaoui, Antoine Jouglet

\section{To cite this version:}

Thierry Denoeux, Nicole El Zoghby, Véronique Cherfaoui, Antoine Jouglet. Optimal object association in the Dempster-Shafer framework. IEEE Transactions on Cybernetics, 2014, 44 (22), pp.2521-2531. 10.1109/TCYB.2014.2309632 . hal-01127786

\section{HAL Id: hal-01127786 \\ https://hal.science/hal-01127786}

Submitted on 9 Mar 2015

HAL is a multi-disciplinary open access archive for the deposit and dissemination of scientific research documents, whether they are published or not. The documents may come from teaching and research institutions in France or abroad, or from public or private research centers.
L'archive ouverte pluridisciplinaire HAL, est destinée au dépôt et à la diffusion de documents scientifiques de niveau recherche, publiés ou non, émanant des établissements d'enseignement et de recherche français ou étrangers, des laboratoires publics ou privés. 


\title{
Optimal object association in the Dempster-Shafer framework
}

\author{
Thierry Denoeux, Nicole El Zoghby, Véronique Cherfaoui, Antoine Jouglet
}

\begin{abstract}
Object association is a crucial step in target tracking and data fusion applications. This task can be formalized as the search for a relation between two sets (e.g., a sets of tracks and a set of observations), in such a way that each object in one set is matched with at most one object in the other set. In this paper, this problem is tackled using the formalism of belief functions. Evidence about the possible association of each object pair, usually obtained by comparing the values of some attributes, is modeled by a Dempster-Shafer mass function defined in the frame of all possible relations. These mass functions are combined using Dempster's rule, and the relation with maximal plausibility is found by solving an integer linear programming problem. This problem is shown to be equivalent to a linear assignment problem, which can be solved in polynomial time using, e.g., the Hungarian algorithm. This method is demonstrated using simulated and real data. The three-dimensional extension of this problem (with three object sets) is also formalized, and is shown to be $\mathcal{N} \mathcal{P}$-hard.
\end{abstract}

Index Terms-Belief functions; Evidence theory; data fusion; assignment problem.

\section{INTRODUCTION}

Object association refers to the task of matching two finite sets of objects $E=\left\{e_{1}, \ldots, e_{n}\right\}$ and $F=\left\{f_{1}, \ldots, f_{p}\right\}$, with possibly different cardinalities. This problem arises, for instance, in multiple target tracking applications [1], [2], in which we need to estimate the status of mobile objects (such as targets or storm cells [3]) that are detected at different times by a single sensor. Data association then consists in deciding which observation should be used to update each track. In this case, the two sets of objects are a set of tracks and a set of observations. Another class of problems in which object association is needed is sensor fusion (see, e.g., [4]). In that case, objects are typically perceived by two sensors and we need to perform observation-to-observation or track-to-track association, depending on the level of sensor outputs. The study of the multi-dimensional case, in which we have more than two sets of objects (perceived, e.g., by several sensors), is deferred until the end of this paper.

Usually, object association is performed under the assumption that each object in one set should be matched with at most one object in the other set. An object in $E$ may have no counterpart in $F$ because it has disappeared between two successive time frames, or because it has not been perceived by one of the sensors. Mathematically, we are thus searching for a relation $R \subseteq E \times F$ such that, for all $i, j$ and $k$ :

$$
\left(e_{i}, f_{j}\right) \in R \text { and }\left(e_{i}, f_{k}\right) \in R \Rightarrow j=k
$$

The authors are with Heudiasyc (UMR 7253), Université de Technologie de Compiègne and CNRS, Compiègne, France. and

$$
\left(e_{i}, f_{k}\right) \in R \text { and }\left(e_{j}, f_{k}\right) \in R \Rightarrow i=j .
$$

Any such relation may be described by a matrix $R$ of size $(n, p)$ such that $R_{i j}=1$ if $\left(e_{i}, f_{j}\right) \in R$ and $R_{i j}=0$ otherwise (by abuse of notation but without any risk of confusion, we use the same notation for the relation and its corresponding matrix).

In this paper, this problem will be investigated in the framework of the theory of belief functions, also referred to as Dempster-Shafer theory or Evidence theory [5]. We assume that we receive evidence about the possible association of each object pair $\left(e_{i}, f_{j}\right)$. Mathematically, such evidence can be represented by a mass function $m_{i j}$ on the frame $\Theta_{i j}=\{0,1\}$, such that $m_{i j}(\{1\})=\alpha_{i j}$ is the probability of knowing that $R_{i j}=1, m_{i j}(\{0\})=\beta_{i j}$ is the probability of knowing that $R_{i j}=0$, and $m_{i j}(\{0,1\})=1-\alpha_{i j}-\beta_{i j}$ is the probability of knowing nothing at all about $R_{i j}$.

Based on such evidence, we would like to choose the "best" relation $R^{*}$, among the set $\mathcal{R}$ of all relations verifying (1a)(1b). The problem of selecting a relation in $\mathcal{R}$ based on pairwise mass functions $m_{i j}$ has been addressed by several authors (see, e.g., [6]-[11]). However, only heuristic solutions have been provided until now. The most elaborate solution so far, proposed by Mercier et al. [10], consists in first combining the mass functions $\left\{m_{i j}\right\}_{j=1}^{p}$ for each $i$, and then finding the relation $R$ with maximum pignistic probability [12]. However, this algorithm involves enumerating all the elements of $R$, which quickly becomes intractable when $n$ and $p$ are not very small. Additionally, the method lacks a fundamental symmetry property, as it may give different results if the sets $E$ and $F$ are interchanged.

In this paper, we show that the above problem, formalized as the search for the most plausible relation $R^{*}$ in $\mathcal{R}$, can be transformed into an equivalent linear assignment problem and solved exactly in polynomial time. The method is studied experimentally using both simulated and real multi-sensor data from an intelligent vehicle application. Finally, we show that the three-dimensional extension of this problem, with three object sets $E, F$ and $G$, is $\mathcal{N} \mathcal{P}$-hard.

The rest of this paper is organized as follows. After recalling the necessary background on the theory of belief functions in Section II, the object assignment problem is formalized and solved in Section III. The sensor fusion application is then addressed in Section IV, where experimental results with simulated and real data are also reported. Finally, the three-dimensional version of the object association problem is studied in Section V and Section VI concludes the paper. 


\section{BACKGROUND ON BELIEF FUNCTIONS}

The theory of belief functions has two main components: equivalent representations of a body of evidence (in the form of mass, belief and plausibility functions), and a combination rule for combining independent items of evidence. These two components are reviewed in Subsection II-A and II-B, respectively.

\section{A. Representation of evidence}

The theory of belief functions is a framework for reasoning under uncertainty based on the modeling of evidence [5]. More precisely, let us assume that we are interested in the value of some variable $\boldsymbol{\theta}$ taking values in a finite domain $\Theta$, called the frame of discernment. Uncertain evidence about $\boldsymbol{\theta}$ may be represented by a (normalized) mass function $m$ on $\Theta$, defined as a function from the powerset of $\Theta$, denoted as $2^{\Theta}$, to the interval $[0,1]$, such that $m(\emptyset)=0$ and

$$
\sum_{A \subseteq \Theta} m(A)=1 .
$$

Each number $m(A)$ is interpreted as a degree of belief attached to the proposition $\boldsymbol{\theta} \in A$ and to no more specific proposition. As argued by Shafer [13], the meaning of such degrees of belief can be better understood by assuming that we have compared our evidence to a canonical chance set-up. The setup proposed by Shafer consists of an encoded message and a set of codes $\Omega=\left\{\omega_{1}, \ldots, \omega_{n}\right\}$, exactly one of which is selected at random. We know the list of codes as well as the chance $p_{i}$ of each code $\omega_{i}$ being selected. Decoding the encoded message using code $\omega_{i}$ produces a message of the form " $\boldsymbol{\theta} \in A_{i}$ " for some $A_{i} \subseteq \Theta$. Then

$$
m(A)=\sum_{\left\{1 \leq i \leq n: A_{i}=A\right\}} p_{i}
$$

is the chance that the original message was " $\boldsymbol{\theta} \in A$ ". Stated differently, it is the probability of knowing that $\theta \in A$. In particular, $m(\Theta)$ is, in this setting, the probability that the original message was vacuous, i.e., the probability of knowing nothing.

The above setting thus consists of a set $\Omega$, a probability measure $\mathbb{P}$ on $\Omega$ and a multi-valued mapping $\Gamma: \Omega \rightarrow 2^{\Theta} \backslash\{\emptyset\}$ such that $A_{i}=\Gamma\left(\omega_{i}\right)$ for each $\omega_{i} \in \Omega$. This is the framework initially considered by Dempster in [14]. The triple $(\Omega, \mathbb{P}, \Gamma)$ formally defines a finite random set [15]. Each piece of evidence can thus be represented by a random set, which induces a mass function.

To each normalized mass function $m$, we may associate belief and plausibility functions from $2^{\Theta}$ to $[0,1]$ defined as follows:

$$
\begin{aligned}
\operatorname{Bel}(A) & =\mathbb{P}(\{\omega \in \Omega \mid \Gamma(\omega) \subseteq A\})=\sum_{B \subseteq A} m(B) \\
P l(A) & =\mathbb{P}(\{\omega \in \Omega \mid \Gamma(\omega) \cap A \neq \emptyset\})=\sum_{B \cap A \neq \emptyset} m(B),
\end{aligned}
$$

for all $A \subseteq \Theta$. These two functions are linked by the relation $\operatorname{Pl}(A)=1-\operatorname{Bel}(\bar{A})$, for all $A \subseteq \Theta$. Each quantity
$\operatorname{Bel}(A)$ may be interpreted as the degree to which the evidence supports $A$, while $\operatorname{Pl}(A)$ can be interpreted as the degree to which the evidence is not contradictory to $A$. The following inequalities always hold: $\operatorname{Bel}(A) \leq \operatorname{Pl}(A)$, for all $A \subseteq \Theta$. The function $p l: \Theta \rightarrow[0,1]$ such that $p l(\theta)=P l(\{\theta\})$ for all $\theta \in \Theta$ is called the contour function associated to $m$.

\section{B. Combination of evidence}

A key idea in Dempster-Shafer theory is that beliefs are elaborated by aggregating different items of evidence. The basic mechanism for evidence combination is Dempster's rule of combination, which can be naturally derived using the random code metaphor as follows. Let $m_{1}$ and $m_{2}$ be two mass functions induced by triples $\left(\Omega_{1}, \mathbb{P}_{1}, \Gamma_{1}\right)$ and $\left(\Omega_{2}, \mathbb{P}_{2}, \Gamma_{2}\right)$ interpreted under the random code framework as before. Let us further assume that the codes are selected independently. For any two codes $\omega_{1} \in \Omega_{1}$ and $\omega_{2} \in \Omega_{2}$, the probability that they both are selected is then $\mathbb{P}_{1}\left(\left\{\omega_{1}\right\}\right) \mathbb{P}_{2}\left(\left\{\omega_{2}\right\}\right)$, in which case we can conclude that $\boldsymbol{\theta} \in \Gamma_{1}\left(\omega_{1}\right) \cap \Gamma_{2}\left(\omega_{2}\right)$. If $\Gamma_{1}\left(\omega_{1}\right) \cap \Gamma_{2}\left(\omega_{2}\right)=\emptyset$, we know that the pair of codes $\left(\omega_{1}, \omega_{2}\right)$ could not have been selected: consequently, the joint probability distribution on $\Omega_{1} \times \Omega_{2}$ must be conditioned, eliminating such pairs [13]. This line of reasoning leads to the following combination rule, referred to as Dempster's rule [5]:

$$
\left(m_{1} \oplus m_{2}\right)(A)=\frac{1}{1-\kappa} \sum_{B \cap C=A} m_{1}(B) m_{2}(C)
$$

for all $A \subseteq \Theta, A \neq \emptyset$ and $\left(m_{1} \oplus m_{2}\right)(\emptyset)=0$, where

$$
\kappa=\sum_{B \cap C=\emptyset} m_{1}(B) m_{2}(C)
$$

is the degree of conflict between $m_{1}$ and $m_{2}$. If $\kappa=1$, there is a logical contradiction between the two pieces of evidence and they cannot be combined. Dempster's rule is commutative, associative, and it admits as neutral element the vacuous mass function defined as $m(\Theta)=1$.

Dempster's rule can be easily expressed in terms of contour functions: if $p l_{1}$ and $p l_{2}$ are the contour functions of two mass functions $m_{1}$ and $m_{2}$, then the contour function of $m_{1} \oplus m_{2}$ is, using the same symbol $\oplus$ as used for mass functions and contour functions:

$$
\left(p l_{1} \oplus p l_{2}\right)(\theta)=\frac{p l_{1}(\theta) p l_{2}(\theta)}{1-\kappa},
$$

for all $\theta \in \Theta$.

\section{OBJECT ASSOCIATION}

In this section, we first show that the problem of finding the most plausible matching between two sets of objects, based on independent evidence pertaining to each pair of objects, can be formalized as a binary linear programming problem (Subsection III-A). We then show in Subsection III-B that this problem is equivalent to a linear assignment problem and we give the complexity of this problem. 


\section{A. Problem formalization}

As explained in Section I, we assume that the available evidence about the association between the sets $E$ and $F$ consists in $n p$ mass functions $m_{i j}, 1 \leq i \leq n, 1 \leq j \leq p$. Each $m_{i j}$ encodes a piece of evidence about a binary variable $R_{i j}$ that equals 1 if $e_{i}$ and $f_{j}$ correspond to the same entity, and 0 otherwise. We note that the absence of information about the association between $e_{i}$ and $f_{j}$ may be encoded by the vacuous mass function such that $m_{i j}(\{0,1\})=1$. Typically, $m_{i j}$ is based on a measure of similarity between some attributes describing the objects. Concrete examples will be discussed in Section IV.

The key idea behind our approach is to express all the available evidence in the frame of discernment $\mathcal{R}$ defined as the set of all possible matchings between $E$ and $F$ verifying (1a)-(1b). Assuming independence between the $n p$ items of evidence, the $n p$ mass functions can then be combined using Dempster's rule (5), and the plausibility of any relation $R \in \mathcal{R}$ may be simply calculated using (7).

Let $\mathcal{R}_{i j}$ denote the set of relations that match objects $e_{i}$ and $f_{j}$ :

$$
\mathcal{R}_{i j}=\left\{R \in \mathcal{R} \mid R_{i j}=1\right\} .
$$

Each mass function $m_{i j}$ on $\Theta_{i j}=\{0,1\}$ may be expressed in $\mathcal{R}$ by transferring the mass $m_{i j}(\{1\})=\alpha_{i j}$ to $\mathcal{R}_{i j}$, $m_{i j}(\{0\})=\beta_{i j}$ to $\overline{\mathcal{R}_{i j}}$ and $m_{i j}(\{0,1\})=1-\alpha_{i j}-\beta_{i j}$ to $\mathcal{R}$, where $\overline{\mathcal{R}_{i j}}$ denotes the complement of $\mathcal{R}_{i j}$. Let $p l_{i j}$ denote the corresponding contour function. It has the following expression:

$$
p l_{i j}(R)= \begin{cases}1-\beta_{i j} & \text { if } R \in \mathcal{R}_{i j}, \\ 1-\alpha_{i j} & \text { otherwise }\end{cases}
$$

for all $R \in \mathcal{R}$, which can be expressed more concisely as follows:

$$
p l_{i j}(R)=\left(1-\beta_{i j}\right)^{R_{i j}}\left(1-\alpha_{i j}\right)^{1-R_{i j}} .
$$

Let $m$ denote the mass function on $\mathcal{R}$ obtained by combining the $n p$ mass using Dempster's rule. From (7), its contour function $p l$ is proportional to the product of the $n p$ mass functions $p l_{i j}$ :

$$
p l(R) \propto \prod_{i, j}\left(1-\beta_{i j}\right)^{R_{i j}}\left(1-\alpha_{i j}\right)^{1-R_{i j}},
$$

and its logarithm is

$$
\begin{aligned}
\ln p l(R)=\sum_{i, j}\left[R_{i j} \ln \left(1-\beta_{i j}\right)+\right. & \\
& \left.\left(1-R_{i j}\right) \ln \left(1-\alpha_{i j}\right)\right]+C,
\end{aligned}
$$

where $C$ is a constant and it is assumed that $\beta_{i j}<1$ and $\alpha_{i j}<1$ for all $i$ and $j$. We note that the situation where $\beta_{i j}=1$ or $\alpha_{i j}=1$ (which corresponds to the case where we have absolute certainty that objects $e_{i}$ and $f_{j}$ should, or should not be matched, respectively) can be easily accounted for by setting $\beta_{i j}=1-\epsilon$ or $\alpha_{i j}=1-\epsilon$ for some arbitrary small $\epsilon>0$.
The most plausible relation $R^{*}$ can thus be found by solving the following linear optimization problem:

$$
\max _{R}\left(\sum_{i, j} w_{i j} R_{i j}\right)
$$

under the constraints (1a)-(1b), with

$$
w_{i j}=\ln \frac{1-\beta_{i j}}{1-\alpha_{i j}} .
$$

Before studying this problem in the next section, we may observe that prior knowledge about the true object association can be easily incorporated in this framework. For instance, assume that relations that match more objects are considered a priori as more plausible or, on the contrary, less plausible. Such prior knowledge can be represented by a belief function with contour function $p l_{0}$ such that:

$$
p l_{0}(R) \propto \exp \left(\lambda \sum_{i, j} R_{i j}\right),
$$

where $\lambda$ is a scalar parameter. A positive (respectively, negative) value of $\lambda$ favors relations $R$ with higher (respectively, lower) cardinality. Combining this contour function with the pairwise contour functions $p l_{i j}$ using Dempster's rule and taking the logarithm, we get

$$
\begin{aligned}
\ln p l(R) \propto \sum_{i, j} & {\left[R_{i j} \ln \left(1-\beta_{i j}\right)+\right.} \\
& \left.\left(1-R_{i j}\right) \ln \left(1-\alpha_{i j}\right)+\lambda R_{i j}\right]+C .
\end{aligned}
$$

The obtained maximization problem would then have the same form as above, with $w_{i j}$ now defined as

$$
w_{i j}=\lambda+\ln \frac{1-\beta_{i j}}{1-\alpha_{i j}} .
$$

\section{B. Problem resolution and complexity analysis}

The problem previously formalized and denoted as $P$ hereafter, can be stated as the following integer linear program:

$$
\max \left(\sum_{i=1}^{n} \sum_{j=1}^{p} w_{i j} R_{i j}\right)
$$

subject to

$$
\begin{array}{lc}
\sum_{j=1}^{p} R_{i j} \leq 1 & \forall i \in\{1, \ldots, n\} \\
\sum_{i=1}^{n} R_{i j} \leq 1 & \forall j \in\{1, \ldots, p\} \\
R_{i j} \in\{0,1\} & \forall i \in\{1, \ldots, n\}, \forall j \in\{1, \ldots, p\},
\end{array}
$$

where constraints (18a) and (18b) are related to Equations (1a) and (1b), respectively.

Without loss of generality, it is considered that $n \geq p$ (if it is not the case, $E$ and $F$ are interchanged). To be solved, $P$ is reduced to the following problem $P^{\prime}$ :

$$
\max \sum_{i=1}^{n} \sum_{j=1}^{n} w_{i j}^{\prime} R_{i j}^{\prime}
$$


subject to

$$
\begin{aligned}
& \sum_{j=1}^{n} R_{i j}^{\prime}=1 \quad \forall i \in\{1, \ldots, n\} \\
& \sum_{i=1}^{n} R_{i j}^{\prime}=1 \quad \forall j \in\{1, \ldots, n\} \\
& R_{i j}^{\prime} \in\{0,1\} \quad \forall i, j \in\{1, \ldots, n\},
\end{aligned}
$$

where $\forall i \in\{1, \ldots, n\}, \forall j \in\{1, \ldots, p\}, w_{i j}^{\prime}=\max \left(0, w_{i j}\right)$ and $\forall i \in\{1, \ldots, n\}, \forall j \in\{p+1, \ldots, n\}, w_{i j}^{\prime}=0$.

Proposition 1. Let $R$ be an optimal solution of problem $P$ and let $R^{\prime}$ be an optimal solution of problem $P^{\prime}$, then $\sum_{i=1}^{n} \sum_{j=1}^{n} w_{i j}^{\prime} R_{i j}^{\prime}=\sum_{i=1}^{n} \sum_{j=1}^{p} w_{i j} R_{i j}$.

Proof: Suppose that

$$
\sum_{i=1}^{n} \sum_{j=1}^{n} w_{i j}^{\prime} R_{i j}^{\prime}<\sum_{i=1}^{n} \sum_{j=1}^{p} w_{i j} R_{i j}
$$

A new solution $R^{\prime \prime}$ for problem $P^{\prime}$ is extracted from solution $R$ in the following way. First, $R^{\prime \prime}$ is initialized with $R_{i j}^{\prime \prime}=$ $0, \forall(i, j) \in\{1, \ldots, n\}^{2}$.

For each $i \in\{1, \ldots, n\}$ such that $\sum_{j=1}^{p} R_{i j}=1, R_{i j}^{\prime \prime}$ is set to $R_{i j}$ for all $j \in\{1, \ldots, p\}$. At this point of the building of solution $R^{\prime \prime}$, note that $\sum_{i=1}^{n} \sum_{j=1}^{n} w_{i j}^{\prime} R_{i j}^{\prime \prime}=$ $\sum_{i=1}^{n} \sum_{j=1}^{p} w_{i j} R_{i j}$. Note also that constraints (20a) and (20b) do not necessarily hold since an object of $E$ has not to be necessarily associated with an object of $F$ in $R^{\prime \prime}$. In this case, there is exactly the same number of indices $i \in\{1, \ldots, n\}$ such that $\sum_{j=1}^{n} R_{i j}^{\prime \prime}=0$ as the number of indices $j \in\{1, \ldots, n\}$ such that $\sum_{i=1}^{n} R_{i j}^{\prime \prime}=0$. Then, each $i \in\{1, \ldots, n\}$ such that $\sum_{j=1}^{n} R_{i j}^{\prime \prime}=0$ is considered iteratively. We search for the smallest indice $j$ with $\sum_{k=1}^{n} R_{k j}^{\prime \prime}=0$ (knowing that this indice necessarily exists) and $R_{i j}^{\prime \prime}$ is set to 1. If $j>p$, then $w_{i j}^{\prime}=0$ by definition of problem $P^{\prime}$. If $j \leq p$, then necessarily $w_{i j}^{\prime}=0$. Indeed, $w_{i j}^{\prime}>0 \Rightarrow w_{i j}>0$ and by setting $R_{i j}$ to 1 (noticing that this new matching can be added to $R$ since $\sum_{k=1}^{p} R_{i k}=\sum_{k=1}^{n} R_{k j}=0$ ), we obtain $a$ new solution for problem $P$ with a higher cost, contradicting that $R$ was optimal. Thus, at the end of each iteration $\sum_{i=1}^{n} \sum_{j=1}^{p} w_{i j}^{\prime} R_{i j}^{\prime \prime}=\sum_{i=1}^{n} \sum_{j=1}^{p} w_{i j} R_{i j}$ still holds. Finally, $R^{\prime \prime}$ is also a solution of $P^{\prime}$ with $\sum_{i=1}^{n} \sum_{j=1}^{p} w_{i j}^{\prime} R_{i j}^{\prime \prime}=$ $\sum_{i=1}^{n} \sum_{j=1}^{p} w_{i j} R_{i j}>\sum_{i=1}^{n} \sum_{j=1}^{p} w_{i j}^{\prime} R_{i j}^{\prime}$ contradicting the fact that $R^{\prime}$ is an optimal solution of $P^{\prime}$.

Now, suppose that $\sum_{i=1}^{n} \sum_{j=1}^{n} w_{i j}^{\prime} R_{i j}^{\prime}>$ $\sum_{i=1}^{n} \sum_{j=1}^{p} w_{i j} R_{i j}$. A new solution $R^{\prime \prime}$ for problem $P$ is extracted from solution $R^{\prime}$ in the following way. For all $i \in\{1, \ldots, n\}$ and for all $j \in\{1, \ldots, p\} R_{i j}^{\prime \prime}$ is set to $R_{i j}^{\prime}$ if $w_{i j} \geq 0$ and to 0 otherwise. Note that $R^{\prime \prime}$ is a solution of $P$ since if constraints (20a) and (20b) hold, then constraints (18a) and (18b) also hold. Since, by definition, $w_{i j}<0 \Rightarrow w_{i j}^{\prime}=0$ then $\sum_{i=1}^{n} \sum_{j=1}^{p} w_{i j} R_{i j}^{\prime \prime}=$ $\sum_{i=1}^{n} \sum_{j=1}^{n} w_{i j}^{\prime} R_{i j}^{\prime}>\sum_{i=1}^{n} \sum_{j=1}^{p} w_{i j} R_{i j}$, contradicting the fact that $R$ is an optimal solution of $P$.

Now, it is clear that an optimal solution for problem $P$ can be obtained by solving problem $P^{\prime}$. Indeed, from any optimal solution $R^{\prime}$ of problem $P^{\prime}$, we can build an optimal solution $R$ for problem $P$ with the same cost value (and then optimal) with $R_{i j}=R_{i j}^{\prime}$ if $w_{i j}>0$ and $R_{i j}=0$ otherwise. Note that building problem $P^{\prime}$ runs in $O\left(n^{2}\right)$ time and that building an optimal solution $R$ of problem $P$ from an optimal solution of problem $P^{\prime}$ runs in $O(n p)$. It now remains to know how to efficiently solve problem $P^{\prime}$.

Fortunately, in the operations research literature, problem $P^{\prime}$ is known as the assignment problem and can be solved in $O\left(n^{3}\right)$ times with the Hungarian Method [16] or in $O\left(n^{5 / 2} \log \left(n \max _{i, j} w_{i j}\right)\right)$ with the algorithm of Orlin and Ahuja [17]. Note that, most of the time, algorithms to solve the assignment problem are described as minimum cost assignment with integer costs. However, the maximization cost problem $P^{\prime}$ can be transformed into a minimization cost problem by taking $\max _{k, \ell} w_{k \ell}-w_{i j}$ instead of the $w_{i j}$ and costs can be transformed into integers by multiplying them by a suitably large number. In practice, since the obtained problem is a special case of the minimum cost flow problem [18], it is possible to use any of the algorithms solving it. In particular, it belongs to a class of integer linear problems for which the constraint matrices are unimodular and can be then solved by a linear programming solver (relaxing Constraints 8 from $R_{i j}^{\prime} \in\{0,1\}$ to $R_{i j}^{\prime} \in[0,1]$ ) knowing that it always has integer solutions.

Example 1. To illustrate the way the object association problem can be transformed into a linear assignment problem, let us consider the following example. Assume that $n=3$, $p=4$, and the $\alpha_{i j}$ s and $\beta_{i j}$ s have the following values:

$$
\begin{gathered}
\left(\alpha_{i j}\right)=\left(\begin{array}{llll}
0.21 & 0.19 & 0.12 & 0.02 \\
0.07 & 0.18 & 0.35 & 0.53 \\
0.52 & 0.27 & 0.49 & 0.40
\end{array}\right) \\
\left(\beta_{i j}\right)=\left(\begin{array}{llll}
0.45 & 0.28 & 0.74 & 0.47 \\
0.34 & 0.42 & 0.31 & 0.39 \\
0.42 & 0.30 & 0.30 & 0.21
\end{array}\right),
\end{gathered}
$$

where the rows and the columns correspond to objects $e_{i}$ and $f_{j}$, respectively. The corresponding weight matrix $W=\left(w_{i j}\right)$ is:

$$
W=\left(\begin{array}{rrrr}
-0.3621 & -0.1178 & -1.2192 & -0.6147 \\
-0.3429 & -0.3463 & 0.0597 & 0.2607 \\
0.1892 & -0.0420 & 0.3167 & 0.2751
\end{array}\right) .
$$

Setting the negative weights to 0 and transforming $W$ into a square matrix, we get

$$
W^{\prime}=\left(\begin{array}{rrrr}
0 & 0 & 0 & 0 \\
0 & 0 & 0.0597 & 0.2607 \\
0.1892 & 0 & 0.3167 & 0.2751 \\
0 & 0 & 0 & 0
\end{array}\right) .
$$

Transforming $W^{\prime}$ to $\max _{k, \ell} w_{k \ell}^{\prime}-W^{\prime}$ in order to define a minimization problem, we have:

$$
W^{\prime \prime}=\left(\begin{array}{llrr}
0.3167 & 0.3167 & 0.3167 & 0.3167 \\
0.3167 & 0.3167 & 0.2570 & 0.0559 \\
0.1274 & 0.3167 & 0 & 0.0416 \\
0.3167 & 0.3167 & 0.3167 & 0.3167
\end{array}\right)
$$


The linear assignment problem with cost matrix $W^{\prime \prime}$ admits the following solution:

$$
R^{\prime}=\left(\begin{array}{llll}
1 & 0 & 0 & 0 \\
0 & 0 & 0 & 1 \\
0 & 0 & 1 & 0 \\
0 & 1 & 0 & 0
\end{array}\right) .
$$

Deleting the last line and setting $R_{i j}=R_{i j}^{\prime}$ if $w_{i j} \geq 0$ and $R_{i j}=0$ otherwise, we get the following final solution:

$$
R=\left(\begin{array}{llll}
0 & 0 & 0 & 0 \\
0 & 0 & 0 & 1 \\
0 & 0 & 1 & 0
\end{array}\right),
$$

meaning that objet $e_{1}$ is not associated, $e_{2}$ is associated with $f_{4}$ and $e_{3}$ is associated with $f_{3}$.

Finally, we can remark that, while the Hungarian algorithm is historically the most popular method to solve the assignment problem, some refinements and generalizations can be found in the literature (see, for example, [2] and [19]), in particular to avoid the transformation to square matrices. These algorithms can be used instead of the proposed method.

\section{SENSOR FUSION APPLICATION}

In this section, we first show how pairwise mass functions can be computed from object attributes in object recognition applications (Subsection IV-A). We then present experimental results with simulated and real data in Subsections IV-B and IV-C, respectively.

\section{A. Computation of mass functions}

Typically, objects are described by a set of attributes. The values of these attributes for each object pair $\left(e_{i}, f_{j}\right)$ can be considered as pieces of evidence regarding the association variable $R_{i j}$. This evidence may be represented as a mass function $m_{i j}$ in different ways, depending on the nature of the attributes. As an illustration, we will consider three attributes commonly used in object recognition applications: position, velocity and class.

Position: Assume that $E$ and $F$ are sets of objects perceived by two sensors, and each sensor also provides an estimated position for each object. Let $d_{i j}$ denote the distance between the estimated positions of $e_{i}$ and $f_{j}$, computed using some distance measure (like the Euclidean distance or the Mahalanobis distance if each sensor also returns a covariance matrix). How can a mass function $m_{i j}$ be deduced from $d_{i j}$ ?

Here, it is clear that a single object cannot have two distinct positions and, conversely, two objects cannot occupy exactly the same position. Consequently, a small value of $d_{i j}$ supports the hypothesis $R_{i j}=1$, while a large value of $d_{i j}$ supports the hypothesis $R_{i j}=0$. Depending on sensor reliability, a fraction of the unit mass should also be assigned to $\Theta_{i j}=\{0,1\}$. This line of reasoning justifies a mass function $m_{i j}^{p}$ of the form:

$$
\begin{aligned}
& m_{i j}^{p}(\{1\})=\rho \varphi\left(d_{i j}\right) \\
& m_{i j}^{p}(\{0\})=\rho\left(1-\varphi\left(d_{i j}\right)\right) \\
& m_{i j}^{p}\left(\Theta_{i j}\right)=1-\rho,
\end{aligned}
$$

where $\rho \in[0,1]$ is a degree of confidence in the sensor information and $\varphi$ is a decreasing function taking values in $[0,1]$. For instance, the following form may be chosen for $\varphi$ :

$$
\varphi(d)=\exp (-\gamma d)
$$

where $\gamma$ is a positive coefficient.

Velocity: Let us now assume that each sensor returns a velocity vector for each object. Let $d_{i j}^{\prime}$ denote the Euclidean distance between the velocity vectors of objects $e_{i}$ and $f_{j}$. Again, $d_{i j}^{\prime}$ is a piece of evidence about $R_{i j}$. However, this piece of evidence does not have the same interpretation as the previous one: here, a large value of $d_{i j}^{\prime}$ supports the hypothesis $R_{i j}=0$, whereas a small value of $d_{i j}^{\prime}$ does not support specifically $R_{i j}=1$ or $R_{i j}=0$, as two distinct objects may have similar velocities. Consequently, the following form of the mass function $m_{i j}^{v}$ induce by $d_{i j}^{\prime}$ seems appropriate:

$$
\begin{aligned}
& m_{i j}^{v}(\{0\})=\rho^{\prime}\left(1-\psi\left(d_{i j}^{\prime}\right)\right) \\
& m_{i j}^{v}\left(\Theta_{i j}\right)=1-\rho^{\prime}\left(1-\psi\left(d_{i j}^{\prime}\right)\right),
\end{aligned}
$$

where, as before, $\rho^{\prime} \in[0,1]$ is a degree of confidence in the sensor information and $\psi$ is a decreasing function taking values in $[0,1]$. This function can be chosen to have the same form as (22), possibly with a different coefficient $\gamma^{\prime}$.

Class: In many applications, objects are categorized in distinct classes such as pedestrian, car, motorcycle, etc. Let $\Omega$ be the set of possible classes, and let $m_{i}$ and $m_{j}$ denote mass functions representing evidence about the class membership of objects $e_{i}$ and $f_{j}$. Such mass functions may be provided, e.g., by evidential pattern classifiers such as described in [20], [21]. Ristic and Smets [22] have considered the object association problem using such class information. However, they made the assumption that equality of class implies equality of objects, a questionable assumption when the number of classes is not much greater than the number of objects. The computation of a mass function $m_{i j}^{\prime \prime}$ on the frame $\Theta_{i j}$ from the mass functions $m_{i}$ and $m_{j}$ on $\Omega$ can be performed rigorously as follows.

Let $S_{i j}$ denote the event that objects $e_{i}$ and $f_{j}$ belong to the same class, and let $\Omega_{i j}=\left\{S_{i j}, \bar{S}_{i j}\right\}$, where $\bar{S}_{i j}$ is the negation of $S_{i j}$. As shown in [23], the belief and plausibility of $S_{i j}$ induced by $m_{i}$ and $m_{j}$ have the following expressions:

$$
\begin{gathered}
\operatorname{Bel}\left(\left\{S_{i j}\right\}\right)=\sum_{\omega \in \Omega} m_{i}(\{\omega\}) m_{j}(\{\omega\})=\eta_{i j}, \\
P l\left(\left\{S_{i j}\right\}\right)=1-\sum_{A \cap B=\emptyset} m_{i}(A) m_{j}(B)=1-\kappa_{i j},
\end{gathered}
$$

where $\kappa_{i j}$ is the degree of conflict (6) between $m_{i}$ and $m_{j}$. The corresponding mass function $\mu_{i j}$ on $\Omega_{i j}$ is

$$
\begin{aligned}
\mu_{i j}\left(\left\{S_{i j}\right\}\right) & =\eta_{i j} \\
\mu_{i j}\left(\left\{\bar{S}_{i j}\right\}\right) & =\kappa_{i j} \\
\mu_{i j}\left(\Omega_{i j}\right) & =1-\eta_{i j}-\kappa_{i j} .
\end{aligned}
$$

Now, it is clear that two objects from different classes cannot be identical, whereas two objects from the same class can be identical or not, which can be formally expressed as follows:

$$
\begin{aligned}
& \bar{S}_{i j} \Rightarrow\left(R_{i j}=0\right) \\
& S_{i j} \Rightarrow\left(R_{i j}=0\right) \text { or }\left(R_{i j}=1\right) .
\end{aligned}
$$


Consequently, a mass function $m_{i j}^{c}$ on $\Theta_{i j}$ can be computed from $\mu_{i j}$ by transferring the mass $\kappa_{i j}$ to $\{0\}$ and the remaining mass $1-\kappa_{i j}$ to $\Theta_{i j}$. We thus get:

$$
\begin{aligned}
& m_{i j}^{c}(\{0\})=\kappa_{i j} \\
& m_{i j}^{c}\left(\Theta_{i j}\right)=1-\kappa_{i j} .
\end{aligned}
$$

For each object pair $\left(e_{i}, f_{j}\right)$, a mass function $m_{i j}$ on $\Theta_{i j}$ representing all the available evidence about $R_{i j}$ can finally be obtained by combining $m_{i j}^{p}, m_{i j}^{v}$ and $m_{i j}^{c}$ using Dempster's rule:

$$
m_{i j}=m_{i j}^{p} \oplus m_{i j}^{v} \oplus m_{i j}^{c} .
$$

\section{B. Simulation experiment}

The above approach to object association was tested using simulated data. Each instance of the assignment problem was randomly generated as follows.

a) Experimental settings: We assumed that each of two agents perceives $n$ objects, of which $80 \%$ are real objects and $20 \%$ are spurious. The position $\mathbf{x}_{i}$ of each real object $i$ was generated from a uniform distribution in the square $[0,5]^{2}$ while its velocity $\mathbf{v}_{i}$ was generated with direction uniformly distributed in $[0,2 \pi)$ and with norm uniformly distributed in $[0,0.5]$. Objects were assumed to belong to one of two equiprobable classes and to be described by a feature $y_{i}$ normally distributed with standard deviation $\sigma=2$ and means -1 in class one and +1 in class two.

For each real object $i$, each of the two agents was assumed to get noisy versions of $\mathbf{x}_{i}, \mathbf{v}_{i}$ and $y_{i}$ defined as follows:

$$
\widehat{\mathbf{x}}_{i}=\mathbf{x}_{i}+\boldsymbol{\epsilon}_{i}, \quad \widehat{\mathbf{v}}_{i}=\mathbf{v}_{i}+\boldsymbol{\epsilon}_{i}^{\prime}, \quad \widehat{y}_{i}=y_{i}+\epsilon_{i}^{\prime \prime},
$$

where $\boldsymbol{\epsilon}_{i}$ and $\boldsymbol{\epsilon}_{i}^{\prime}$ are Gaussian noises with mean $(0,0)$ and variance $0.04 I$ ( $I$ being the identity matrix) and $\epsilon_{i}^{\prime \prime}$ is a Gaussian noise with mean 0 and standard deviation 0.2. Mass functions $m_{i j}^{p}$ and $m_{i j}^{v}$ were computed as described in Subsection IV-A with the Euclidean distance. Each agent was also assumed to compute a mass function $m_{i}$ about the class of object $i$ from its noisy feature $\widehat{y}_{i}$ using the following formula:

$$
m_{i}(\{1\})=\frac{f_{1}\left(\widehat{y}_{i}\right)}{f_{1}\left(\widehat{y}_{i}\right)+f_{2}\left(\widehat{y}_{i}\right)}, \quad m_{i}(\{2\})=1-m_{i}(\{1\}),
$$

where $f_{k}$ is the density distribution of $y_{i}$ in class $k$.

The spurious objects were generated as real ones, but independently for the two agents. An example of such an association problem with $n=40$ objects (including 32 "real" ones) is represented in Figure 1.

b) Performance assessment: The number $n$ of objects was varied between 5 and 80 . For each value of $n, 30$ association problems were generated and solved using the algorithm described in Section III, with $\rho=\rho^{\prime}=0.7, \gamma=\gamma^{\prime}=0.2$ and $\lambda=0$. The quality of the association was measured by two criteria: precision and recall defined, respectively, as the fraction of matched pairs that are correct, and the fraction of true object pairs that were matched. Figure 2 shows the mean precision and recall as functions of $n$ in three cases: using the position information only, using position and velocity, and using all three sources of information (position, velocity and class). As expected, the method makes effective use of

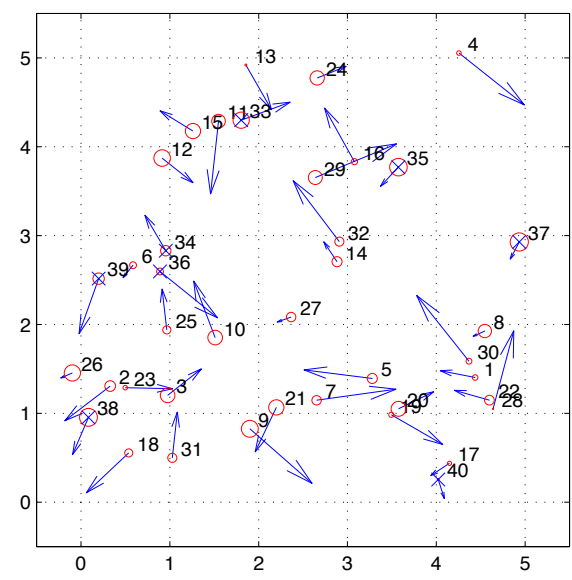

(a)

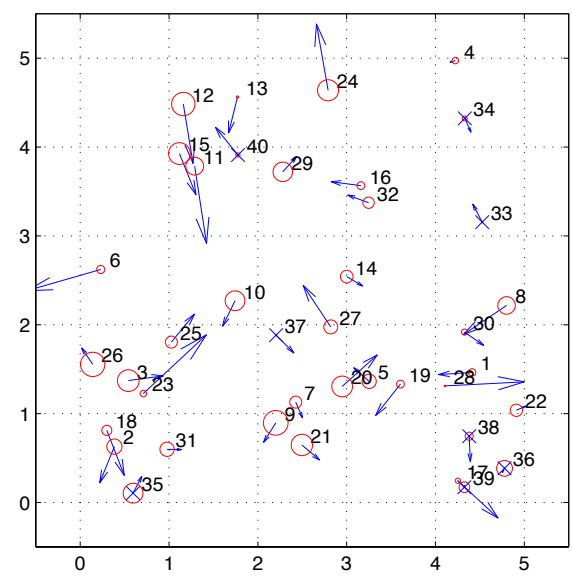

(b)

Fig. 1. Example of an association problem with 40 objects perceived by 2 sensors ( $\mathrm{a}$ and $\mathrm{b}$ ). Objects 33 to 40 (marked by a $\mathrm{x}$ ) are spurious. The size of the circles is proportional to the mass $m_{i}(\{1\})$ of class 1 .

additional information encoded in the pairwise mass functions, and the performances degrade gracefully with $n$. Figure 3 shows the mean computing times as a function of $n$; the association algorithm was programmed in Matlab and run on a Macbook Pro personal computer.

c) Influence of $\rho$ and $\gamma$ : We studied the influence of parameters $\rho\left(=\rho^{\prime}\right)$ and $\gamma\left(=\gamma^{\prime}\right)$ on the quality of the association. For each pair $(\rho, \gamma)$, we computed the F-measure, defined as the harmonic mean between precision and recall:

$$
F=2 \frac{\text { precision } \cdot \text { recall }}{\text { precision }+ \text { recall }} \text {. }
$$

Parameter $\lambda$ was set to 0 . All three sources of information (position, velocity and class) were used. For each pair $(\rho, \gamma)$, the values of the F-measure were averaged over 30 association problems with $n=20$ objects. The results are reported in Table I. As we can see, the choice of parameters $\rho$ and $\gamma$ does influence the results, and the best performances were obtained for $\rho=0.7$ and $\gamma=0.2$. These results show that parameters $\rho$ 


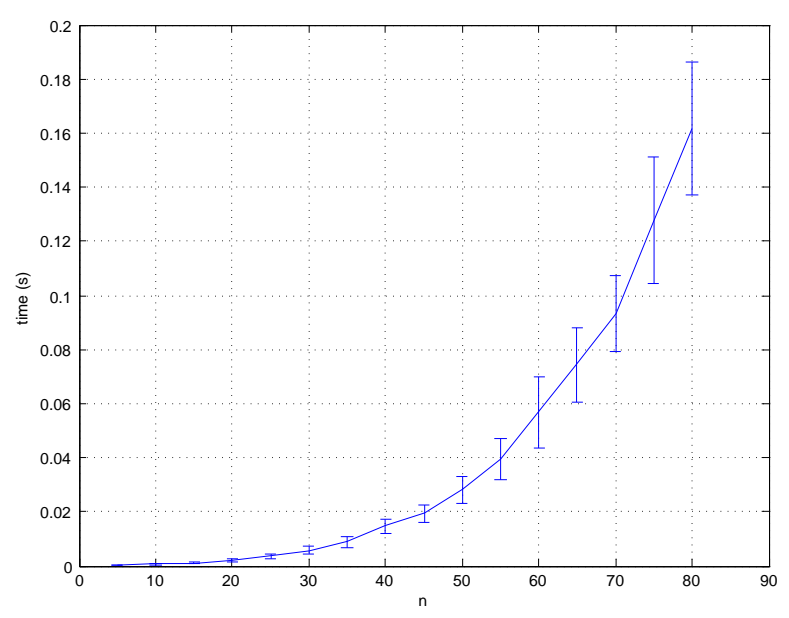

Fig. 3. Mean running time (in seconds) plus or minus one standard deviation, as a function of $n$.

and $\gamma$ do have an influence on the quality of the association. If a dataset with matched objects is available, the performances of the system can be significantly enhanced by learning these parameters from the data.

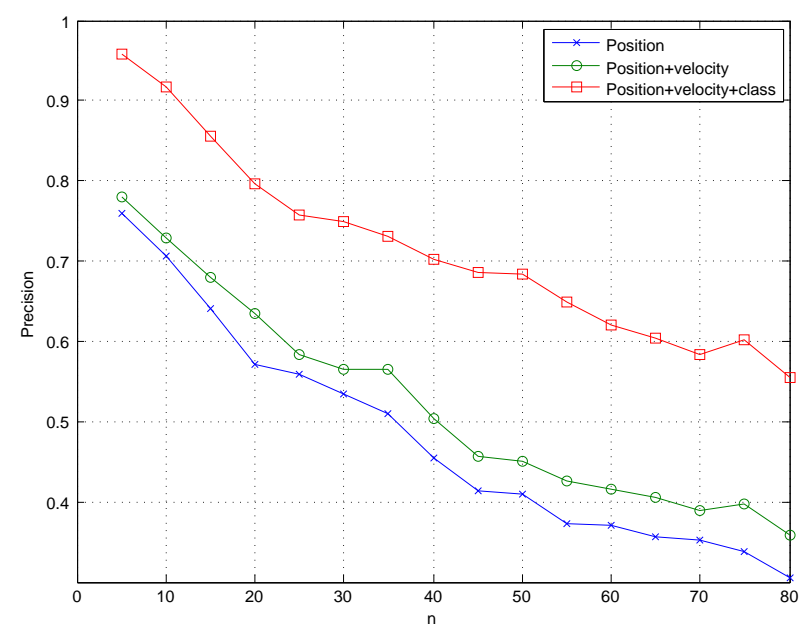

(a)

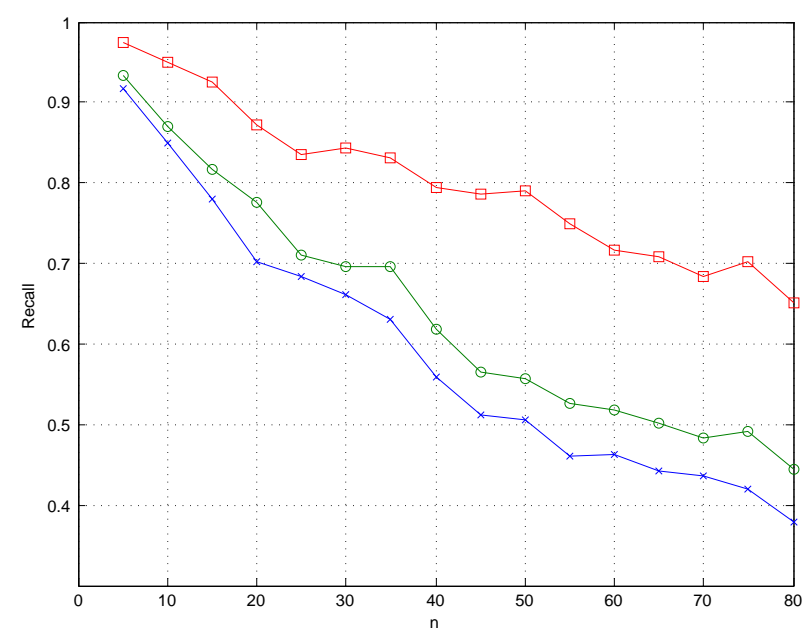

(b)

Fig. 2. Mean precision (a) and recall (b) as a function of $n$.
TABLE I

F-MEASURE FOR DIFFERENT VALUES OF $\rho$ AND $\gamma$ (AVERAGES OVER 30 ASSOCIATION PROBLEMS WITH $n=20$ OBJECTS, TAKING INTO ACCOUNT POSITION, VELOCITY AND CLASS).

\begin{tabular}{cc|ccccc} 
& & & & & $\gamma$ & \\
& & 0.1 & 0.2 & 0.3 & 0.4 & 0.5 \\
\hline \multirow{4}{*}{$\rho$} & 0.5 & 0.641 & 0.559 & 0.490 & 0.425 & 0.326 \\
& 0.6 & 0.836 & 0.842 & 0.744 & 0.666 & 0.587 \\
& 0.7 & 0.795 & $\underline{0.858}$ & 0.847 & 0.797 & 0.722 \\
& 0.8 & 0.770 & 0.829 & 0.846 & 0.838 & 0.798 \\
& 0.9 & 0.750 & 0.804 & 0.826 & 0.827 & 0.821
\end{tabular}

d) Influence of $\lambda$ : We also studied the influence of $\lambda$ in (14) and (16). We recall that this parameter makes it possible to introduce prior knowledge about the number of associations, with positive (respectively, negative) favoring larger (respectively, smaller) numbers of matched object pairs. Consequently, increasing $\lambda$ can be expected to increase recall (as more true object pairs will be matched) but to decrease precision (as more incorrect pairs will also be matched). An experiment was carried out with $n=40$ objects generated as explained above, with $\lambda$ ranging between -1 and +3 . For each value of $\lambda, 200$ association problems were generated. 


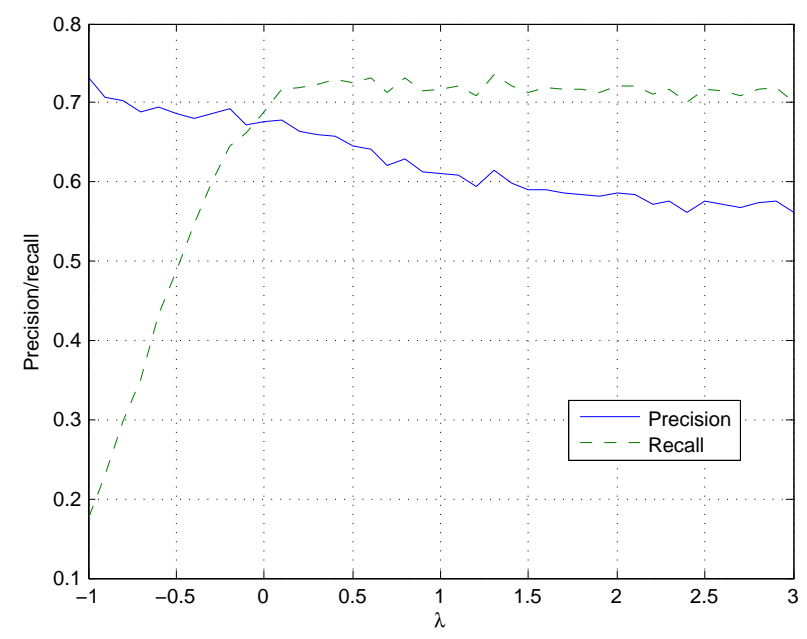

Fig. 4. Mean precision and recall (averaged over 200 association problems with $n=40$ objects), as a function of $\lambda$.

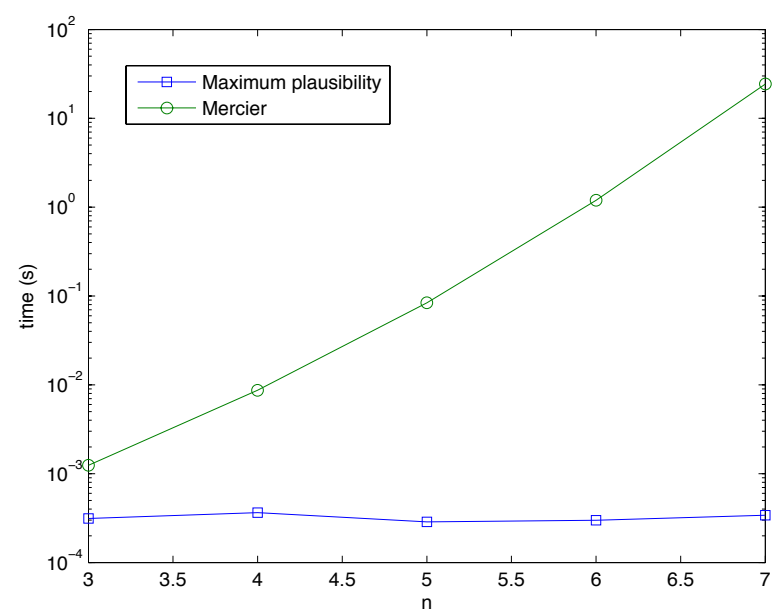

Fig. 5. Mean running time (in seconds) for our method (maximum plausibility) and Mercier's algorithm, as a function of $n$.

The results reported in Figure 4 confirm that different values $\lambda$ indeed result in different trade-offs between precision and recall. However, a higher precision can only be obtained at the cost of a very low recall for that problem. By assigning different weights to precision and recall, it would be possible to find an optimal value of $\lambda$. In most applications, however, it might be sufficient to set $\lambda=0$ as a reasonable default value.

e) Comparison with Mercier's method: Finally, our algorithm was compared to Mercier's method [10]. However, the comparison could only be carried for small values of $n$ $(n \leq 7)$ because the computational complexity of this method restricts its applications to very small numbers of objects. On this problem, both methods yield identical solutions in almost all cases. However, as shown in Figure 5, the running time of Mercier's method grows exponentially with $n$, whereas the time complexity of our method is polynomial.

\section{Experiment with real data}

Our approach was applied as part of an advanced driver assistance application [24]. A car was equipped with two sensors: a vision-based Mobileye system and a four-layer Ibeo Alasca-XT Laser scanner. Each sensor has an associated information processing system allowing it to track and classify objects. It is thus a track-to-track association problem [2] but we do not use the history of the tracks (or their identity) to match them.

For each sensor, position-based mass functions $m_{i j}^{p}$ were computed using (21) and (22) with $\rho=0.9, \gamma=0.1$ and $d_{i j}$ defined as the Mahalanobis distance:

$$
d_{i j}=\sqrt{\left(\mathbf{x}_{i}-\mathbf{x}_{j}\right)^{\prime}\left(P_{i}+P_{j}\right)^{-1}\left(\mathbf{x}_{i}-\mathbf{x}_{j}\right)},
$$

where $\mathbf{x}_{i}$ denotes the estimated position of the center of object $i$ and $P_{i}$ is the estimated covariance matrix of the estimate.

Each sensor also predicts the class of objects, expressed in different frames. The laser classifies the objects as pedestrian or non pedestrian using the algorithm developed by Fayad et al. [24]; this algorithm computes a mass function over the two classes. The Mobileye system has a finer frame of discernment with five classes: pedestrian, car, truck, motorbike, bicycle. As the latter sensor provides a predicted class, but no confidence in the prediction, a mass 0.9 was assigned to the predicted class, and 0.1 to the whole frame of discernment. For each pair of mass functions $m_{i}$ and $m_{j}$, a mass function $m_{i j}^{c}$ was computed based on their degree of conflict using (27), and it was combined with $m_{i j}^{p}$ using Dempster's rule. We note that velocity information was not used in this application.

The considered dataset contains 58 frames (association problems). The Mobileye sensor detected between 1 and 3 objects (average: 2) while the laser scanner wass less selective and detected between 2 and 23 objects (average: 8.5). An example of an association problem is shown in Figures 6a and $6 \mathrm{~b}$, with corresponding result shown in Figure 6c. The average precision and recall for our algorithm (with $\lambda=0$ ) were 0.75 and 0.90 , respectively (with standard deviations 0.33 and 0.30). Mercier's algorithm achieved identical results, with considerably longer computing time (119 seconds on average, against 0.23 seconds for our method).

\section{THREE-DIMENSIONAL EXTENSION}

As an extension of the problem addressed in this paper, we consider in this section the situation where we have more than two sets of objects. To keep the notation simple, and without loss of generality, we will consider the three-dimensional association problem, in which we have three sets of objects. The problem will first be formalized in Subsection V-A and its complexity will then be studied in Subsection V-B.

\section{A. Formalization}

Let $E=\left\{e_{1}, \ldots, e_{n}\right\}, F=\left\{f_{1}, \ldots, f_{p}\right\}$ and $G=$ $\left\{g_{1}, \ldots, g_{q}\right\}$ denote the three sets of objects perceived, e.g., by three sensors. We are now searching for three relations $R \subset E \times F, S \subset F \times G$ and $T \subset E \times G$ representing, respectively, the correspondence between objects in sets $E$ and 
$F, F$ and $G, E$ and $G$. Each of these three relations has to verify properties (1a)-(1b). Let $\mathcal{R}, \mathcal{S}$ and $\mathcal{T}$ denote the sets of relations $R, S$ and $T$ verifying these properties. Additionally, relations $R, S$ and $T$ cannot be determined independently: for instance, if $\left(e_{i}, f_{j}\right) \in R$ and $\left(f_{j}, g_{k}\right) \in S$, then we must have $\left(e_{i}, g_{k}\right) \in T$. More generally, for any three objects $(e, f, g) \in E \times F \times G$, if any two pairs of objects are related, then the third pair has to be related too. Formally, the three following implications must hold for any triplet $(i, j, k)$ :

$$
\begin{aligned}
& \left(e_{i}, f_{j}\right) \in R \text { and }\left(f_{j}, g_{k}\right) \in S \Rightarrow\left(e_{i}, g_{k}\right) \in T \\
& \left(e_{i}, f_{j}\right) \in R \text { and }\left(e_{i}, g_{k}\right) \in T \Rightarrow\left(f_{j}, g_{k}\right) \in S \\
& \left(f_{j}, g_{k}\right) \in S \text { and }\left(e_{i}, g_{k}\right) \in T \Rightarrow\left(e_{i}, f_{j}\right) \in R .
\end{aligned}
$$

The set of solutions to the association problem is thus the set $\mathcal{U}$ of triplets $(R, S, T) \in \mathcal{R} \times \mathcal{S} \times \mathcal{T}$ verifying (33).

As before, we shall assume that we receive pieces of

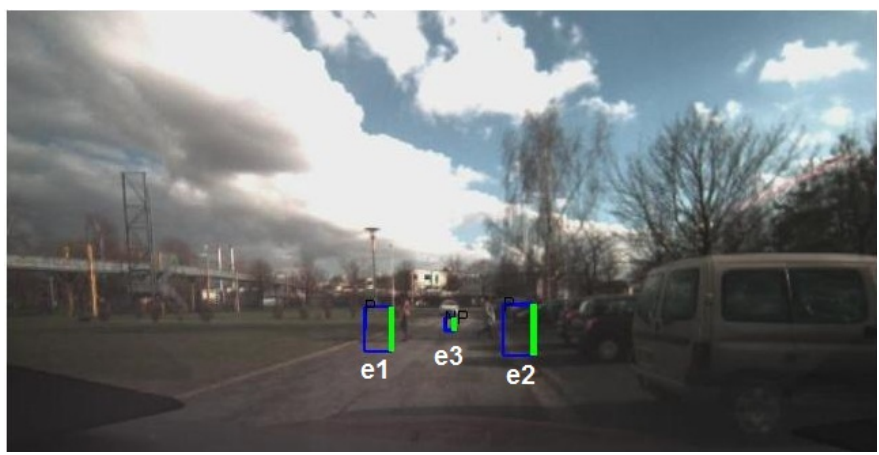

(a)

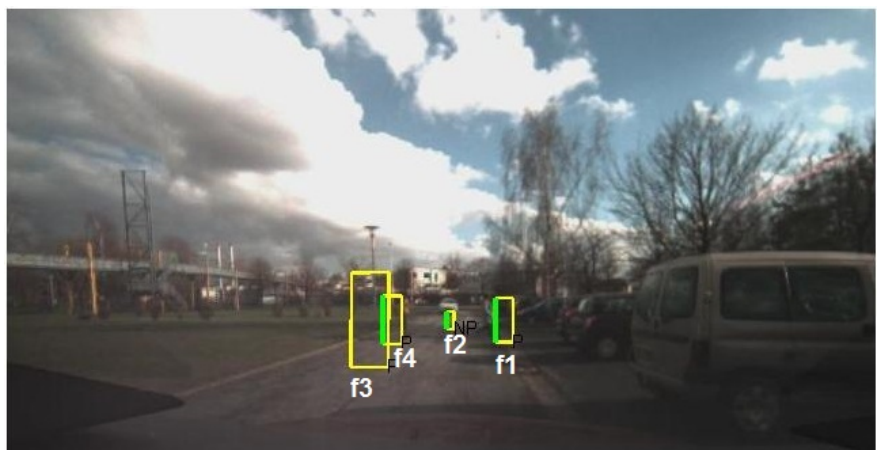

(b)

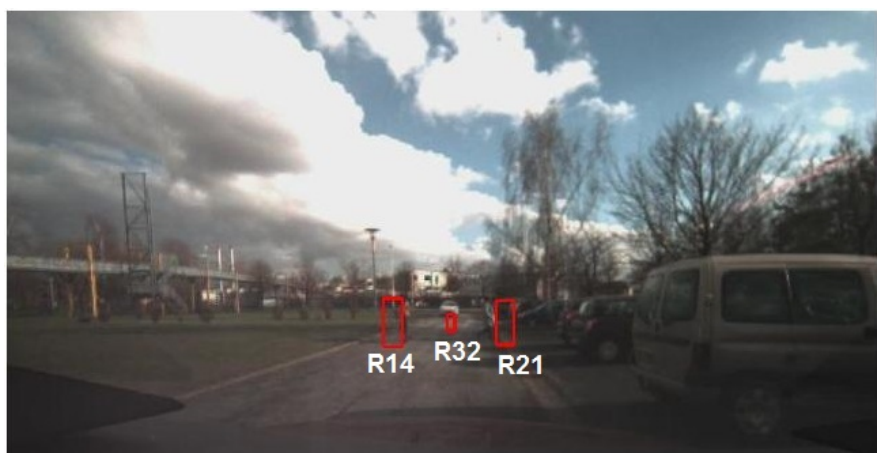

(c)

Fig. 6. Example of an association problem: the laser sensor detects three objects (a) while the Mobileye sensor detects four objects, including a spurious one (b). The association algorithm correctly matches the three real objects (c). evidence regarding the association of any pair of objects in $E \times F, F \times G$ and $E \times G$, and these pieces evidence are encoded as pairwise mass functions $m_{i j}$., $m_{\cdot j k}$ and $m_{i \cdot k}$, for all $i, j$ and $k$. As before, these mass functions need to be expressed in the common frame of discernment $\mathcal{U}$ before being combined by Dempster's rule.

Let $U_{i j}$. denote the set of triplets $(R, S, T) \in \mathcal{U}$ such that $R_{i j}=1$, and let $m_{i j} .(\{1\})=\alpha_{i j}$. and $m_{i j} .(\{0\})=\beta_{i j .}$. To express $m_{i j}$. in $\mathcal{U}$, we need to transfer the mass $\alpha_{i j}$. to $U_{i j}$., $\beta_{i j}$. to $\overline{U_{i j}}$. and $1-\alpha_{i j}$. $-\beta_{i j}$. to $\mathcal{U}$. The corresponding contour function in $\mathcal{U}$ is then defined as follows:

$$
\operatorname{pl}_{i j .}(R, S, T)= \begin{cases}1-\beta_{i j} . & \text { if } R_{i j}=1, \\ 1-\alpha_{i j} . & \text { otherwise }\end{cases}
$$

for all $(R, S, T) \in \mathcal{U}$, which can be expressed more concisely 
as follows:

$$
p l_{i j} .(R, S, T)=\left(1-\beta_{i j} .\right)^{R_{i j}}\left(1-\alpha_{i j} .\right)^{1-R_{i j}} .
$$

Similarly, with obvious notations, mass functions $m_{\cdot j k}$ and $m_{i \cdot k}$ induce the following contour functions in $\mathcal{U}$ :

$$
\begin{aligned}
& p l_{\cdot j k}(R, S, T)=\left(1-\beta_{\cdot j k}\right)^{S_{j k}}\left(1-\alpha_{\cdot j k}\right)^{1-S_{j k}} \\
& p l_{i \cdot k}(R, S, T)=\left(1-\beta_{i \cdot k}\right)^{T_{i k}}\left(1-\alpha_{i \cdot k}\right)^{1-T_{i k}} .
\end{aligned}
$$

Combining the $n p+p q+n q$ pieces of evidence by Dempster's rule yields the following contour function:

$$
\begin{aligned}
p l(R, S, T) \propto \prod_{i, j, k}\left[\left(1-\beta_{i j} \cdot\right)^{R_{i j}}\right. & \\
\left(1-\alpha_{i j \cdot}\right)^{1-R_{i j}} & \left(1-\beta_{\cdot j k}\right)^{S_{j k}}\left(1-\alpha_{\cdot j k}\right)^{1-S_{j k}} \\
& \left.\left(1-\beta_{i \cdot k}\right)^{T_{i k}}\left(1-\alpha_{i \cdot k}\right)^{1-T_{i k}}\right] .
\end{aligned}
$$

Taking the logarithm and, as before, assuming the $\alpha$ s and $\beta$ s to be strictly smaller than one, we get:

$$
\begin{aligned}
\ln p l(R, S, T)= & \sum_{i, j} w_{i j} \cdot R_{i j}+ \\
& \sum_{j, k} w_{\cdot j k} S_{j k}+\sum_{i, k} w_{i \cdot k} T_{i k}+C,
\end{aligned}
$$

where $C$ is a constant and

$$
\begin{gathered}
w_{i j .}=\ln \frac{1-\beta_{i j .}}{1-\alpha_{i j .}}, \quad w_{\cdot j k}=\ln \frac{1-\beta_{\cdot j k}}{1-\alpha_{\cdot j k}}, \\
w_{i \cdot k}=\ln \frac{1-\beta_{i \cdot k}}{1-\alpha_{i \cdot k}} .
\end{gathered}
$$

The most plausible association $\left(R^{*}, S^{*}, T^{*}\right)$ can thus be found by solving the following binary linear programming problem:

$$
\max _{R, S, T}\left(\sum_{i, j} w_{i j} \cdot R_{i j}+\sum_{j, k} w_{\cdot j k} S_{j k}+\sum_{i, k} w_{i \cdot k} T_{i k}\right)
$$

subject to

$$
\begin{aligned}
\sum_{j=1}^{p} R_{i j} \leq 1, \quad \sum_{i=1}^{n} R_{i j} \leq 1 & \forall(i, j) \\
\sum_{j=1}^{p} S_{j k} \leq 1, \quad \sum_{k=1}^{q} S_{j k} \leq 1 & \forall(j, k) \\
\sum_{k=1}^{q} T_{i k} \leq 1, \quad \sum_{i=1}^{n} T_{i k} \leq 1 & \forall(i, k) \\
R_{i j}+S_{j k} \leq T_{i k}+1 & \forall(i, j, k) \\
R_{i j}+T_{i k} \leq S_{j k}+1 & \forall(i, j, k) \\
S_{j k}+T_{i k} \leq R_{i j}+1 & \forall(i, j, k) \\
R_{i j} \in\{0,1\}, S_{j k} \in\{0,1\}, T_{i k} \in\{0,1\} & \forall(i, j, k)
\end{aligned}
$$

where constraints (41d)-(41f) ensure property (33).

In the special case where all mass functions in one of the three sets $\left\{m_{i j \cdot}\right\},\left\{m_{\cdot j k}\right\}$ or $\left\{m_{i \cdot k}\right\}$ are vacuous, the problem becomes very simple. For instance, assume that all mass functions $m_{i \cdot k}$ are vacuous. This is the case when, for instance, $E, F$ and $G$ are sets of objects perceived by a single sensor at successive time frames, and we only compute the similarity between objects perceived at two consecutive times. We then have $w_{i \cdot k}=0$ for all $i$ and $k$ and the objective function (40) becomes a function of $R$ and $S$ only. As constraints (41d)-(41f) can always be satisfied for some relation $T$, given $R$ and $S$, the objective function can be maximized with respect to $R$ and $S$ separately. In that special case, the three-dimensional problem can thus be solved by solving two two-dimensional problems of the form studied in Section III. In the general case, however, the three-dimensional problem is much harder than the two-dimensional one, as will be shown in the next subsection.

\section{B. Complexity analysis}

Just as the search for the most plausible relation $R^{*}$ in $\mathcal{R}$ is very close to the linear assignment problem, finding the most plausible association $\left(R^{*}, S^{*}, T^{*}\right) \in \mathcal{U}$ is close to a problem known as the axial 3-dimensional assignment problem (3DAP) [25], which is $\mathcal{N} \mathcal{P}$-Hard in the general case. However, several special classes of the problem are polynomially solvable [26]. 3DAP can be seen as a special case of our three-dimensional extension with $|E|=|F|=|G|=n$ and where $R^{*}, S^{*}, T^{*}$ are all bijective relations. It is equivalent to finding exactly $n$ triplets $(e, f, g) \in E \times F \times G$ which are pairwise disjoints. The major difference with our problem is that, when searching for the most plausible association $\left(R^{*}, S^{*}, T^{*}\right) \in \mathcal{U}$, we can have $R_{i j}^{*}=1$ while there is no $k$ such that $S_{j k}^{*}=1$ and $T_{i k}^{*}=1$.

Keep in mind that even if we manage to find a transformation of the most plausible 3-dimensional association problem to 3DAP, it does not mean that we have direct methods to solve our problem (since 3DAP is $\mathcal{N} \mathcal{P}$-Hard), neither does it mean that our problem is $\mathcal{N} \mathcal{P}$-Hard itself. Unfortunately, it turns out to be the case, as proved in the following proposition.

Proposition 2. The most plausible 3-dimensional association problem is $\mathcal{N} \mathcal{P}$-Hard.

Proof: To show this result, we use a reduction of the pairwise consistent 3-dimensional matching problem [27] denoted as PC 3DM in the following and which is $\mathcal{N} \mathcal{P}$-complete:

INSTANCE: Set $M \subseteq W \times X \times Y$, where $W, X$ and $Y$ are disjoint sets having the same number $r$ of elements. $M$ is pairwise consistent, i.e., for all elements $a, b, c$, whenever there exists elements $w, x$ and $y$ such that $(a, b, y) \in M$, $(a, x, c) \in M$, and $(w, b, c) \in M$, then $(a, b, c) \in M$.

QUESTION: Does $M$ contain a subset $M^{\prime} \subseteq M$ such that $\left|M^{\prime}\right|=r$ and no two elements of $M^{\prime}$ agree in any coordinate ?

With a given instance of $P C 3 D M$, we associate an instance of the 3-dimensional association problem in the following way. We take $E=W, F=X$ and $G=Y$ and then $n=p=q=r$. For all $\left(e_{i}, f_{j}, g_{k}\right) \in M$, we set $w_{i j} .=w_{i \cdot k}=w_{\cdot j k}=1$, while all other weights are set to 0 . Note that this reduction is polynomial. We consider the decision version of the 3-dimensional association problem, in which the question is whether there exists a 3-dimensional association $(R, S, T) \in \mathcal{U}$ such that the value of the objective function is $3 r$.

Suppose that there exists a subset $M^{\prime} \subseteq M$ such that $\left|M^{\prime}\right|=r$ and no two elements of $M^{\prime}$ agree in any coordinate. 
For each $\left(e_{i}, f_{j}, g_{k}\right) \in M^{\prime}$, we set $R_{i j}=S_{i k}=T_{j k}=1$ while all other values defining the relations $R, S$ and $T$ are set to 0 . By construction, such relations respect constraints (33) and the value of the objective function is $3 r$ since all the $3 r$ weights associated with the pairs which are kept in the relations have value 1.

Now, suppose that there exists a 3-dimensional association $(R, S, T) \in \mathcal{U}$ such that the value of the objective function is $3 r$. Therefore, each of the $r$ objects $e_{i} \in E$ is associated with exactly one object $f_{j} \in F\left(e_{i} R f_{j}\right)$ with $w_{i j} .=1$ and exactly one objet $g_{k}$ in $G\left(e_{i} T g_{k}\right)$ with $w_{i \cdot k}=1$ such that $f_{j} S g_{k}$ holds and $w_{\cdot j k}=1$. For each of the $r$ such associations $e_{i}, f_{j}, g_{k}$ defined by these relations we have $\left(e_{i}, f_{j}, g_{k}\right) \in M$. Indeed, $w_{i j} .=1$ implies that $\exists y \in Y=G$ such that $\left(e_{i}, f_{j}, y\right) \in M, w_{i \cdot j}=1$ implies that $\exists x \in X=F$ such that $\left(e_{i}, x, g_{k}\right) \in M, w_{\cdot j k}=1$ implies that $\exists w \in W=E$ such that $\left(w, e_{i}, g_{k}\right) \in M$, and finally it implies that $\left(e_{i}, f_{j}, g_{k}\right) \in M$ since $M$ is pairwise consistent. Thus, there exists a subset $M^{\prime} \subseteq M$ such that $\left|M^{\prime}\right|=r$ and no two elements of $M^{\prime}$ agree in any coordinate.

Thus, it is unlikely to solve the 3-dimensional association problem in polynomial time. As future research, we can adapt some methods of the literature for 3DAP to solve our problem. We can then consider branch-and-bound approaches (see, for example, [28]) or heuristic algorithms (such as described in [29]) if the problem is too hard to solve from an operational point of view.

\section{CONCLUSION}

Object association is an important problem in a wide range of applications and a key component of many data fusion systems. Belief functions have often been considered as a convenient formalism for representing and combining information in multi-sensor applications (see, e.g., [10], [11], [22], [30]). However, previous attempts to solve the assignment problem within the Dempster-Shafer framework had led until now to ad hoc and sometimes very time-consuming methods (see, e.g., [6], [8]-[11]).

In this paper, evidence about the possible association of any two pairs of objects has been modeled by DempsterShafer mass functions defined over the frame of all possible relevant relations between the two object sets. The plausibility of any single relation after pooling all available mass functions can then be computed efficiently and maximized to find the most plausible relation. This problem has been shown to be equivalent to a linear assignment problem, which can be solved in polynomial time using, e.g., the Hungarian algorithm. This solution is thus both optimal and computationally much more efficient than previous approaches to this problem in the belief function framework [10].

As an extension of this work, the three-dimensional association problem has also been considered. This problem occurs, e.g., when fusing reports from three sensors. Although this problem can be formalized in the same way as the two-dimensional one, it was shown to be $\mathcal{N} \mathcal{P}$-hard, which makes it unlikely that an optimal solution can be found in polynomial time. The development of heuristic methods to find approximate solutions to this problem is left for further research.

\section{ACKNOWLEDGMENT}

This work was carried out in the framework of the Labex MS2T, which was funded by the French Government, through the program "Investments for the future" managed by the National Agency for Research (Reference ANR-11-IDEX0004-02). The authors would like to thank G. Dherbomez for his help with the acquisition and preprocessing of the experimental data used in Section IV-C, and Vincent Frémont who developed the tools that allowed us to exploit the data from the Mobileye system.

\section{REFERENCES}

[1] Y. Bar-Shalom, T. Fortmann, Tracking and data association, Academic Press, Boston, 1988.

[2] S. Blackman, R. Popoli, Design and Analysis of Modern Tracking Systems, Artech House, 1999.

[3] T. Einfalt, T. Denœux and G. Jacquet, "A radar rainfall forecasting method designed for hydrological purposes," Journal of Hydrology, vol. 114, pp. 229-244, 1990.

[4] A. Houenou, Ph. Bonnifait, V. Cherfaoui and J. F. Boissou, "A TrackTo-Track Association Method for Automotive Perception Systems", In IEEE Intelligent Vehicles Symposium, Alcala de Henares, Spain, 3-7 June 2012, pp. 704-710.

[5] G. Shafer, A mathematical theory of evidence. Princeton, N.J.: Princeton University Press, 1976.

[6] M. Rombaut and V. Cherfaoui, "Decision making in data fusion using Dempster-Shafer's theory," in 3th IFAC Symposium on Intelligent Components and Instrumentation for Control Applications, Annecy, France, 9-11 june 1997, pp 375-379.

[7] D. Gruyer and V. Cherfaoui, "Matching and decision for vehicle tracking in road situation," in IEEE/RSJ International Conference on Intelligent Robots and Systems IROS'99, Kyongju, Korea, 17-21 October 1999, vol.1, pp. 29-34.

[8] D. Gruyer, C. Royère, R. Labayrade, and D. Aubert, "Credibilistic multisensor fusion for real time application, application to obstacle detection and tracking," in IEEE Int. Conf. on Advanced Robotics, ICAR'2003, Coimbra, Portugual, June 30-July 3 2003, pp. 1462-1467.

[9] N. Megherbi, S. Ambellouis, O. Colot, and F. Cabestaing, "Multimodal data association based on the use of belief functions for multiple target tracking," in 8th International Conference on Information Fusion (FUSION), Philadelphia, PA (USA), 2005, pp. 900-906.

[10] D. Mercier, E. Lefèvre, and D. Jolly, "Object association with belief functions, an application with vehicles," Information Sciences, vol. 181, no. 24 , pp. 5485-5500, 2011.

[11] A. Dallil, M. Oussalah, and A. Ouldali, "Sensor fusion and target tracking using evidential data association," IEEE sensors journal, vol. 13, no. 1, pp. 285-293, 2013.

[12] P. Smets and R. Kennes. The Transferable Belief Model. Artificial Intelligence, 66(2):191-243, 1994.

[13] G. Shafer, "Constructive probability," Synthese, vol. 48, no. 1, pp. 1-60, 1981.

[14] A. P. Dempster, "Upper and lower probabilities induced by a multivalued mapping," Annals of Mathematical Statistics, vol. 38(2), pp. 325-339, 1967.

[15] H. Nguyen, An Introduction to Random Sets. Boca Raton, Florida: Chapman and Hall/CRC Press, 2006.

[16] H. Kuhn, "The Hungarian method for the assignment problem," Naval Research Logistics Quarterly, vol. 2(1-2), pp. 83-97, 1955.

[17] J. Orlin and R. Ahuja, "New scaling algorithms for the assignment and minimim cycle mean problems," Mathematical programming, vol. 54(1), pp. 41-56, 1992.

[18] R. Ahuja, T. Magnanti, and J. Orlin, Network flows: theory, algorithms, and applications. Prentice-Hall, 1993.

[19] F. Bourgeois and J.-C. Lassalle, "An extension of the Munkres algorithm for the assignment problem to rectangular matrices," Communication $A C M$, vol. 14 , no. 12 , pp. 802-804, 1971. 
[20] T. Denœux, "A $k$-nearest neighbor classification rule based on Dempster-Shafer theory," IEEE Trans. on Systems, Man and Cybernetics, vol. 25, no. 05, pp. 804-813, 1995.

[21] _ "A neural network classifier based on Dempster-Shafer theory," IEEE Trans. on Systems, Man and Cybernetics A, vol. 30, no. 2, pp. 131-150, 2000.

[22] B. Ristic and P. Smets, "Global cost of assignment in the TBM framework for association of uncertain ID reports," Aerospace Science and Technology, vol. 11(4), pp. 303-309, 2007.

[23] T. Denœux and M.-H. Masson, "EVCLUS: Evidential clustering of proximity data," IEEE Trans. on Systems, Man and Cybernetics B, vol. 34, no. 1, pp. 95-109, February 2004.

[24] F. Fayad, V. Cherfaoui, and G. Derbhomez, "Updating confidence indicators in a multi-sensor pedestrian tracking system," in IEEE Intelligent Vehicles Symposium (IV 2008), Eindhoven, The Netherlands, 2008, pp 156-161.

[25] E. Schell, "Distribution of a product by several properties, directorate of management analysis," in proceedings of the Second Symposium in Linear Programming. DCS/Comptroller H.Q. U.S.A.F., Washington, DC., 1955, pp. 615-642.

[26] R. Burkard and E. Çela, Handbook of Combinatorial Optimization Supplement Volume A. Kluwer Academic Publishers, 1999, ch. Linear Assignment Problems and Extensions.

[27] M. Garey and D. Johnson, Computers and intractability: a guide to the theory of NP-completeness. W.H. Freeman, 1979.

[28] E. Balas and M. Saltzman, "An algorithm for the three-index assignment problem," Operations Research, vol. 39(1), pp. 150-161, 1991.

[29] W. Pierskalla, "The tri-substitution method for the threemultidimensional assignment problem," Canadian ORS Journal, vol. 5, pp. 71-81, 1967.

[30] J. Schubert. "Clustering decomposed belief functions using generalized weights of conflict", International Journal of Approximate Reasoning, vol. 48, no. 2, pp. 466-480, 2008.

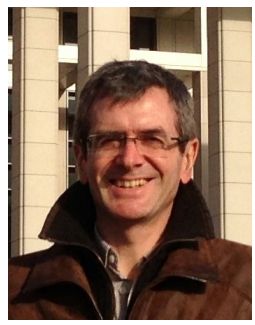

Thierry Denoux Thierry Denoeux graduated in 1985 as an engineer from the Ecole des Ponts ParisTech in Paris, and received a doctorate from the same institution in 1989. Currently, he is Full Professor (Classe Exceptionnelle) with the Department of Information Processing Engineering at the Université de Technologie de Compiègne (UTC), France, and deputy director of the Heudiasyc research Lab (UMR 7253). His research interests concern the management of uncertainty in intelligent systems. His main contributions are in the theory of belief functions with applications to pattern recognition, data mining and information fusion. He is the Editor-in-Chief of the International Journal of Approximate Reasoning, and an Associate Editor of several journals including Fuzzy Sets and Systems and IEEE Transactions on Fuzzy Systems.

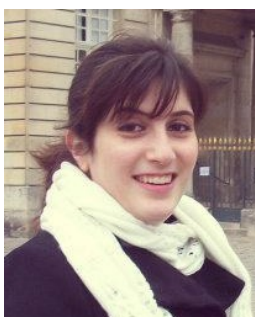

Nicole El Zoghby Nicole El Zoghby received an Electrical Engineering degree from Lebanese University, Faculty of engineering in 2009 and a M.S. degree in Engineering of complex systems from Université Henri Poincaré Nancy, France. She is currently a $\mathrm{PhD}$ student at the Heudiasyc research Lab, working on distributed data fusion in VANETS.

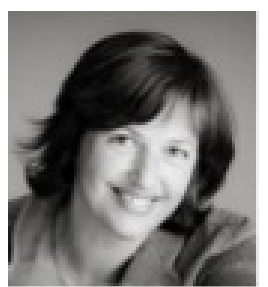

Véronique Cherfaoui Véronique Cherfaoui received the M.S. degree in computer science from the University of Lille, France, in 1988 and a Ph.D. degree in control of systems from the Université de Technologie de Compiègne (UTC), France in 1992. She defended the "Habilitation à Diriger les Recherches" in 2009. She is now an Associate Professor with the Department of Information Processing Engineering at UTC and a member of the Heudiasyc research Lab. Her research interests include multi-sensor data fusion, distributed data fusion, data association and real-time perception systems for intelligent vehicles.

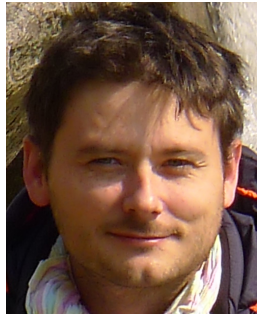

Antoine Jouglet Antoine Jouglet is an Associate Professor with the Heudiasyc research Lab (CNRS) of Université de Technologie de Compiègne (UTC), France. He is also member of the Laboratory of Excellence (Labex) Control of Technological Systemsof-Systems (MS2T). He received the Ph.D degree in Control of Systems from UTC in 2002. His research interests concern combinatorial optimization. He has published several papers on scheduling, warehouse management, organization and bin packing problems. He is member of the Société Française de Recherche Opérationnelle et d'Aide à la Décision (ROADEF). 